\title{
Natural convection melting of nano-enhanced phase change material in a cavity with finned copper profile
}

\author{
Nadezhda Bondareva, and Mikhail Sheremet* \\ Laboratory on Convective Heat and Mass Transfer, Tomsk State University, 36 Lenin Avenue, 634050 Tomsk, Russia
}

\begin{abstract}
Present study is devoted to numerical simulation of heat and mass transfer inside a cooper profile filled with paraffin enhanced with $\mathrm{Al}_{2} \mathrm{O}_{3}$ nanoparticles. This profile is heated by the heat-generating element of constant volumetric heat flux. Two-dimensional approximation of melting process is described by the Navier-Stokes equations in non-dimensional variables such as stream function, vorticity and temperature. The enthalpy formulation has been used for description of the heat transfer. The influence of volume fraction of nanoparticles and intensity of heat generation on melting process and natural convection in liquid phase has been studied.
\end{abstract}

\section{Introduction}

Cooling and maintenance of temperature has a significant role in electronic industry and power engineering. During the operating of the semiconductor appliances and accumulators a large amount of energy generates. To dissipate this energy the metal profiles with high thermal conductivity are used. The presence of fins on the profile increases heat exchange with the environment.

Paraffin is widely used in application in the field of heat storage and energy absorption. Phase transition is accompanied by either absorption or release of energy. As a rule, paraffin waxes has a low melting temperature and it is suitable for operating of electronic systems. Many experimental and numerical researches are devoted to melting of paraffin. Phase change materials such as paraffin wax in spite of high latent heat have a relatively low thermal conductivity. Therefore, it can be used in conjunction with metal profiles, metal foams, graphite matrixes and others. One of the ways to enhance heat transfer inside the system containing paraffin is the adding of nanoparticles to paraffin $[1,2]$. Including of nanoparticles with high heat conductivity allows to increase the intensity of heat exchange [3-6]. It was shown experimentally that thermal conductivity increases with a growth of loadings of nanoparticles and temperature $[3,5]$. Bayat et al. analyzed numerically heat transfer inside the heat sink filled with PCM enhanced by nanoparticles of aluminum oxide and cooper oxide [7]. The authors showed that the volume fraction of nanoparticles is reflected in time of reaching critical temperature. The liquid volume fraction rises faster with adding nanoparticles.

At the same time the effect of particles on the viscosity of the suspension and influence of nanoparticles size play an important role in the fluid motion and phase transition [8]. The combination of these factors determines the heat transfer modes inside the material. Numerical investigations of melting process of ice with $\mathrm{Cu}$ nanoparticles inside in the thermal storage unit were carried out by Jourabian et al. $[9,10]$. The domains under consideration represent a twodimensional cross-section model of heated tube with constant temperature inside round [9] or square [10] shells. It was shown that a growth of particle volumetric concentration reduces the influence of heater position, due to a raise of viscosity and as a consequence due to the weakening of convection heat transfer. It should be noted that for the case of low Rayleigh numbers $\left(R a=10^{5}\right.$ and $\left.R a=2 \cdot 10^{5}\right)$ the addition of nanoparticles leads to an intensification of melting in the lower part of the region and has little effect on the melting rate at the top $[9,10]$, whereas in the case of high Rayleigh number $R a=1.25 \cdot 10^{7}$ the melting rate rises both above the heater and under the heater [11].

Another factor affecting the heat transfer is the change in the latent energy of suspension melting. An inclusion of solid particles of greater density leads to a reduction of the latent heat.

Present study is devoted to a numerical investigation of heat transfer inside the passive cooling system based on nano-enhanced phase-change material.

\section{Physical and mathematical model}

Figure 1 shows the geometry of considered twodimensional domain. It represents a rectangular cavity with a copper radiator filled with pure n-octadecane, the bottom wall of which is in contact with a rectangular source of constant volumetric heat generation. The heater has thermophysical properties of silicon. The initial temperature of the whole system is the same as the ambient temperature. Heat exchange with the

*Corresponding author: Michael-sher@yandex.ru 
environment is carried out through the upper wall of the region, other borders of the system are considered to be thermally insulated. Fluid motion is assumed to be laminar. The thermophysical properties of paraffin, such as thermal conductivity, viscosity and density both in solid paraffin and in melt are considered to be constant and independent of temperature. The Boussinesq approximation for describing the buoyancy force effect inside the molten region, is applied.

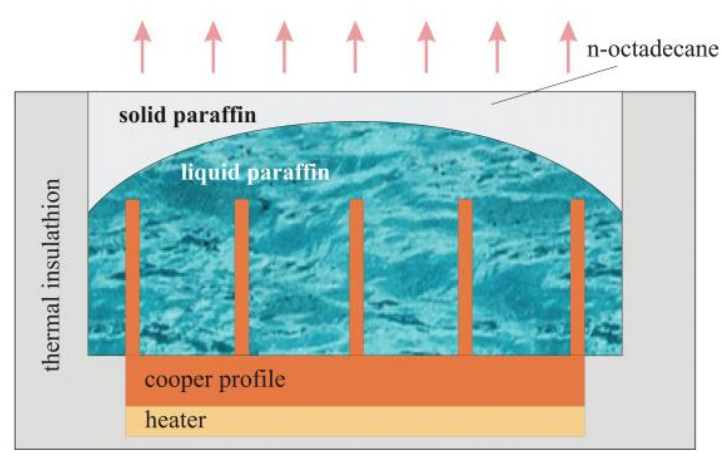

Fig. 1. Physical model.

Governing equations in primitive variables velocity and pressure can be written as:

$$
\begin{gathered}
\frac{\partial u}{\partial x}+\frac{\partial v}{\partial y}=0 \\
\frac{\partial u}{\partial t}+u \frac{\partial u}{\partial x}+v \frac{\partial u}{\partial y}=-\frac{1}{\rho_{n m}} \frac{\partial p}{\partial x}+\frac{\mu_{n m}}{\rho_{n m}}\left(\frac{\partial^{2} u}{\partial x^{2}}+\frac{\partial^{2} u}{\partial y^{2}}\right) \\
\frac{\partial v}{\partial t}+u \frac{\partial v}{\partial x}+v \frac{\partial v}{\partial y}=-\frac{1}{\rho_{n m}} \frac{\partial p}{\partial y}+\frac{\mu_{n m}}{\rho_{n m}}\left(\frac{\partial^{2} v}{\partial x^{2}}+\frac{\partial^{2} v}{\partial y^{2}}\right)+ \\
+\frac{(\rho \beta)_{n m}}{\rho_{n m}} g\left(T-T_{m}\right)
\end{gathered}
$$

The heat conduction equation within the solid phase and energy equation inside the liquid phase of paraffin can be written as follows:

$$
\begin{gathered}
\frac{\partial h}{\partial t}=\left(k_{s}\right)_{n m}\left(\frac{\partial^{2} T}{\partial x^{2}}+\frac{\partial^{2} T}{\partial y^{2}}\right) \\
\frac{\partial h}{\partial t}+u \frac{\partial h}{\partial x}+v \frac{\partial h}{\partial y}=\left(k_{l}\right)_{n m}\left(\frac{\partial^{2} T}{\partial x^{2}}+\frac{\partial^{2} T}{\partial y^{2}}\right)
\end{gathered}
$$

For the heat source and the copper profile, the heat transfer can be described by the following heat conduction equations:

$$
\begin{gathered}
\rho_{1} c_{1} \frac{\partial T}{\partial t}=k_{1}\left(\frac{\partial^{2} T}{\partial x^{2}}+\frac{\partial^{2} T}{\partial y^{2}}\right) \\
\rho_{2} c_{2} \frac{\partial T}{\partial t}=k_{2}\left(\frac{\partial^{2} T}{\partial x^{2}}+\frac{\partial^{2} T}{\partial y^{2}}\right)+Q
\end{gathered}
$$

In present work, the hydrodynamics equations were solved using the stream function and vorticity variables defined as: $u=\partial \psi / \partial y, \quad v=-\partial \psi / \partial x \quad$ and $\omega=\partial v / \partial x-\partial u / \partial y$. Dimensionless variables were correlated to dimensional ones through the relations:

$$
\begin{gathered}
X=x / H, Y=y / H, U=u / V_{0}, V=v / V_{0}, \\
V_{0}=\sqrt{g \beta\left(T_{h}-T_{F}\right) H}, \tau=H / V_{0}, \Omega=\omega H / V_{0}, \\
\Theta=\left(T-T_{F}\right) /\left(T_{h}-T_{F}\right), \Psi=\psi /\left(V_{0} H\right)
\end{gathered}
$$

Taking into account these relations (8) the governing equations (1)-(5) can be written as follows:

$$
\begin{gathered}
\frac{\partial^{2} \Psi}{\partial X^{2}}+\frac{\partial^{2} \Psi}{\partial Y^{2}}=-\Omega \\
\frac{\partial \Omega}{\partial \tau}+U \frac{\partial \Omega}{\partial X}+V \frac{\partial \Omega}{\partial Y}=\frac{\mu_{n m} / \mu_{f}}{\rho_{n m} / \rho_{f}} \sqrt{\frac{P r}{R a}} \nabla^{2} \Omega+ \\
+\frac{(\rho \beta)_{n m} /(\rho \beta)_{f}}{\rho_{n m} / \rho_{f}} \frac{\partial \Theta}{\partial X} \\
\zeta(\varphi)\left[\frac{\partial \Theta}{\partial \tau}+U \frac{\partial \Theta}{\partial X}+V \frac{\partial \Theta}{\partial Y}\right]+\frac{\rho_{n m} L_{n m}}{\rho_{f} L_{f}} \cdot S t e \cdot\left[\frac{\partial \varphi}{\partial \tau}+\right. \\
\left.+U \frac{\partial \varphi}{\partial X}+V \frac{\partial \varphi}{\partial Y}\right]=\frac{\xi(\varphi)}{\sqrt{R a \cdot P r}} \nabla^{2} \Theta
\end{gathered}
$$

In energy equation the smoothing function $\varphi$, reflecting the melt fraction, and the auxiliary functions $\zeta(\varphi)$ and $\xi(\varphi)$ were introduced:

$$
\begin{gathered}
\varphi= \begin{cases}0, & T<T_{m}-\eta \\
\frac{T-\left(T_{m}-\eta\right)}{2 \eta}, & T_{m}-\eta \leq T \leq T_{m}+\eta \\
1, & T>T_{m}+\eta\end{cases} \\
\zeta(\varphi)=\frac{\left(\rho_{s} c_{s}\right)_{n m}}{\rho_{l} c_{l}}+\varphi\left(\frac{\left(\rho_{l} c_{l}\right)_{n m}}{\rho_{l} c_{l}}-\frac{\left(\rho_{s} c_{s}\right)_{n m}}{\rho_{l} c_{l}}\right), \\
\xi(\varphi)=\frac{\left(k_{s}\right)_{n m}}{k_{l}}+\varphi\left(\frac{\left(k_{l}\right)_{n m}}{k_{l}}-\frac{\left(k_{s}\right)_{n m}}{k_{l}}\right)
\end{gathered}
$$

Dimensionless energy equation for the heater can be written as:

$$
\frac{\partial \Theta}{\partial \tau}=\frac{\alpha_{2} / \alpha_{0}}{\sqrt{R a \cdot P r}}\left(\nabla^{2} \Theta+O s\right)
$$

Here the Ostrogradsky number $(O s)$ characterizes the intensity of volumetric heat generation of the heater.

The thermophysical properties of the nanomaterial are determined using the following correlations [7]: 


$$
\begin{aligned}
& (\rho \beta)_{n m}=(1-\Phi)(\rho \beta)_{f}+\Phi(\rho \beta)_{n p} \\
& (\rho c)_{n m}=(1-\Phi)(\rho c)_{f}+\Phi(\rho c)_{n p} \\
& \rho_{n m}=(1-\Phi) \rho_{f}+\Phi \rho_{n p} \\
& c_{n m}=(\rho c)_{n m} / \rho_{n m}
\end{aligned}
$$

The correlation for the effective thermal conductivity can be taken for the liquid paraffin as [5]:

$$
\begin{aligned}
& k_{n m}=\frac{k_{n p}+2 k_{f}-2\left(k_{f}-k_{n p}\right) \Phi}{k_{n p}+2 k_{f}+\left(k_{f}-k_{n p}\right) \Phi} k_{f}+ \\
& +5 \cdot 10^{4} \beta_{\lambda} \Phi \rho_{f} c_{f} \sqrt{\frac{\kappa T}{\rho_{n p} d_{n p}}} f(T, \Phi)
\end{aligned}
$$

and in the solid material:

$$
k_{n m}=\frac{k_{n p}+2 k_{m}-2\left(k_{f}-k_{n p}\right) \Phi}{k_{n p}+2 k_{m}+\left(k_{f}-k_{n p}\right) \Phi} k_{m}
$$

Here

$$
\beta_{\lambda}=8.4407(100 \Phi)^{-1.07304}
$$
$\kappa=1.381 \cdot 10^{-23} \mathrm{~J} / \mathrm{K}$ is the Boltzmann constant, the function $f(T, \Phi)$ determines as:

$$
\begin{aligned}
& f(T, \Phi)=\left(2.817 \cdot 10^{-2} \Phi+3.917 \cdot 10^{-3}\right) \frac{T}{T_{0}}+ \\
& +\left(-3.0669 \cdot 10^{-2} \Phi-3.91123 \cdot 10^{-3}\right)
\end{aligned}
$$

Here $T_{0}=273 \mathrm{~K}$ and $T=320 \mathrm{~K}$, latent heat is $L_{n m}=\frac{(1-\Phi) \rho_{m} L_{m}}{\rho_{n m}}$.

The nanofluid viscosity was defined as

$$
\mu_{n m} / \mu_{f}=0.983 \exp (12.959 \Phi)
$$

The dimensionless parameters were defined as $\operatorname{Pr}=v \rho_{l} c_{l} / k_{l}$ is the Prandtl number; Ste $=L_{f} /\left(c_{l} \Delta T\right)$ is the Stefan number; $R a=g \beta \Delta T H^{3} /\left(v \alpha_{l}\right)$ is the Rayleigh number; $O s=q H^{2} /\left(k_{2} \Delta T\right)$ is the Ostrogradsy number; $B i=\bar{h} H / k$ is the Biot number.

\subsection{Initial and boundary conditions}

At the initial time, the temperature of system was set to equal the ambient temperature $\Theta=\Theta_{0}$.

Boundary conditions for the governing equations are:

- condition at the interface between the copper profile and the phase change material

$$
\Theta_{m}=\Theta_{1}, k_{m} \frac{\partial \Theta_{m}}{\partial Y}=k_{1} \frac{\partial \Theta_{1}}{\partial Y}
$$

- condition at the interface between the copper profile and the heat source:

$$
\Theta_{1}=\Theta_{2}, k_{1} \frac{\partial \Theta_{1}}{\partial Y}=k_{2} \frac{\partial \Theta_{2}}{\partial Y}
$$

- the convective boundary condition is set at the upper border

$$
\frac{\partial \Theta}{\partial Y}=-B i\left(\Theta-\Theta_{0}\right)
$$

- Adiabatic boundary conditions for temperature were set at other borders:

$$
\frac{\partial \Theta}{\partial \bar{n}}=0
$$

On the solid boundaries of the area occupied by the melted paraffin the no-slip boundary conditions have been used for velocity in the form: $\Psi=0, \Omega=-\nabla^{2} \Psi$.

\subsection{Numerical procedure}

To solve the dimensionless equations (9)-(11) with corresponding initial and boundary conditions (20)-(23) the finite difference method was used [12-14]. The difference elliptic equation for stream function was solved by using the successive over relaxation method. The energy and vorticity equations were solved by using the Samarskii's local one-dimensional scheme. This algorithm was verified and the results were in good agreement with experimental and numerical data of other authors.

\section{Results and Discussion}

Figure 2 shows isotherms for different nanoparticles volume fractions and dimensionless time. It can be seen that at initial stage of heating (upper row) the presence of nanoparticles promotes the rapid melting of the paraffin, taking into account the phase front position. The movement of the interphase occurs mostly due to heat conduction. At the same time, a growth of time (bottom row) leads to more intensive melting in the case of low nanoparticles concentration due to convective flow intensification, while a rise of nanoparticles volume fraction results in a growth of the melt viscosity and as a result convective flow attenuates. Therefore, for high $\Phi$ one can find less intensive melting. 
$a$
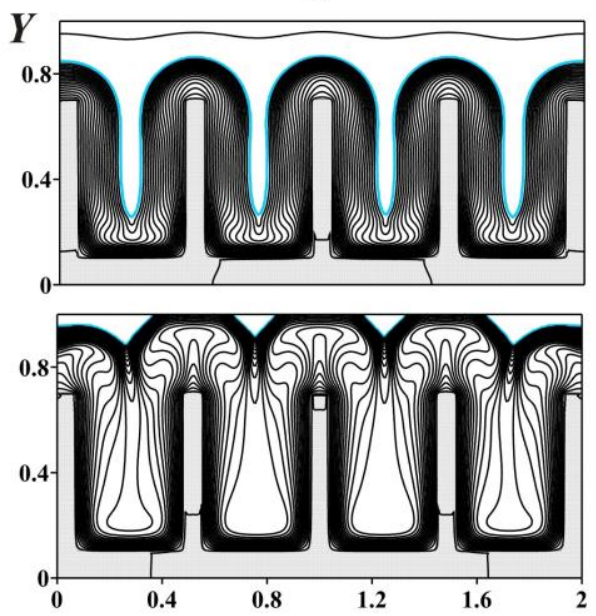

$b$
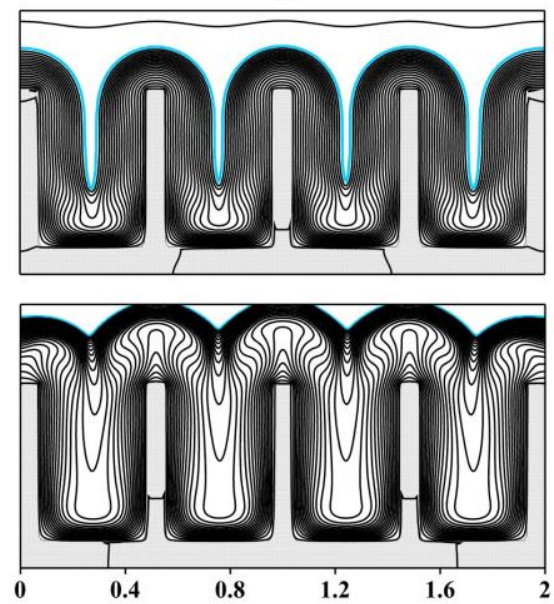

$c$
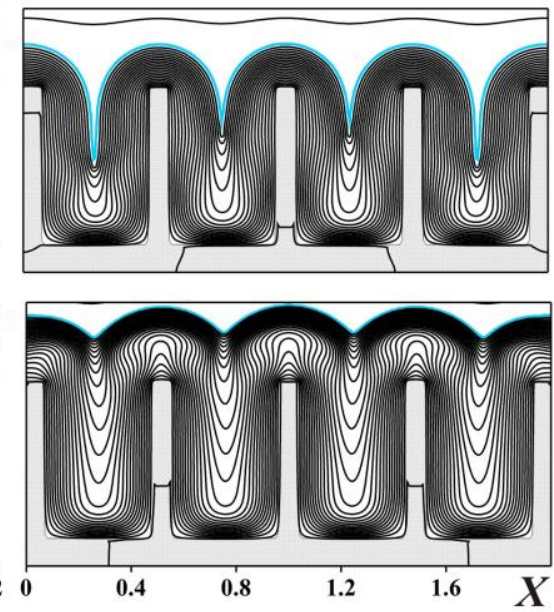

Fig. 2. Isotherms for $O s=0.007$ at $\tau=636.3$ (upper row) and $\tau=848.4$ (bottom row): $a$ ) $\Phi=0, b) \Phi=0.04, c) \Phi=0.08$.

Figure 3 presents the mean Nusselt number time curves. Initial increase in the average Nusselt number relates with heating of the cavity due to heat conduction. As soon as the temperature of profile surface reaches the fusion temperature the melting process begins with absorbing a large amount of energy. The Nusselt number is growing rapidly, until the entire fins surface is covered by a layer of melt. Further, the phase front is removed from the copper profile, where temperature gradually increases, the mean Nusselt number is changing insignificantly. With the appearance and development of natural convection, the interphase boundary moves upward, vertical velocity increases and the upper part of the melt region warms up faster, while at the bottom part the descending flow of the liquid cools the spaces between the fins.

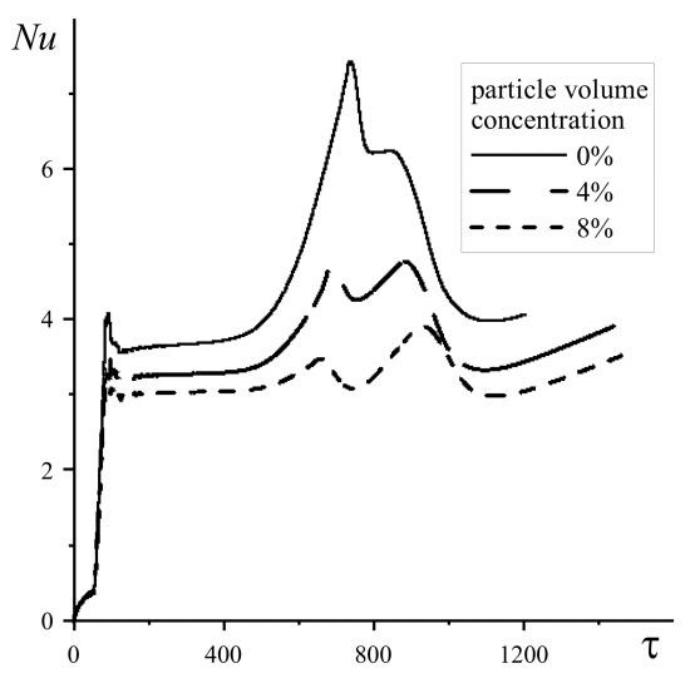

Fig. 3. Nusselt number time curves for $O s=7 \cdot 10^{-3}$.

When convective mode is formed in the melt, the cold flows fall down, as a result the cooling of the plate surface and increasing the heat transfer are occurs. With adding of nanoparticles, this effect becomes less pronounce. Enlargement of viscosity in nano-enhanced material leads to weakening of convective flows. The development of the circulation flow with the expansion of the melt region promotes melting, with addition of particles and an increase in viscosity, the melting rate decreases.

Figure 4 shows the evolution of the liquid volume fraction. An inclusion of nanoparticles inside the PCM results in more intensive melting of the medium at the initial and middle level of the process, while in the case of whole melting an addition of nanoparticles rise the time for complete melting.

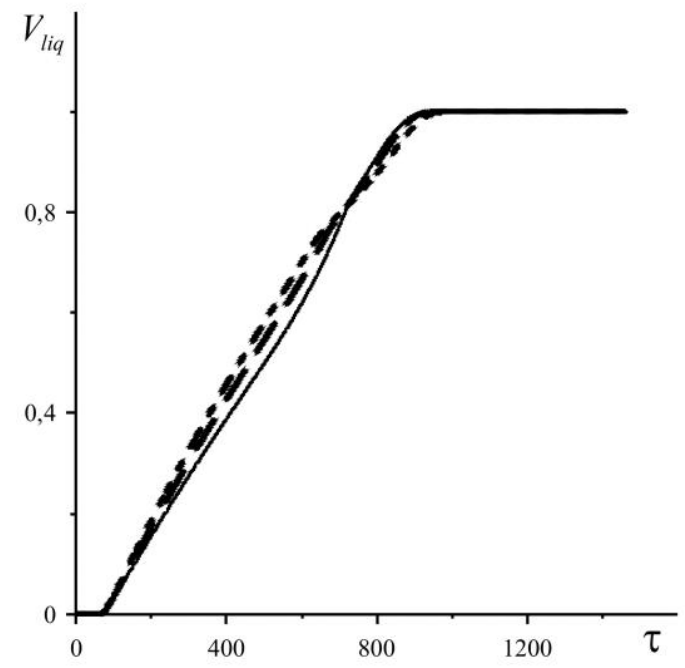

Fig. 4. Liquid volume fraction time curves for $O s=7 \cdot 10^{-3}$.

\section{Conclusions}

Numerical investigation of conjugate natural convection during the melting process of nano-enhanced phase change material inside the cooling system with heat sink was carried out. Distributions of isotherms were received for different nanoparticles volume fraction and dimensionless time. It was shown that an increase in the nanoparticles concentration leads to the melting process accelerates at the initial and middle level of the considered process due to a rise of the thermal conductivity, while with the growth of the melt volume and the development of natural convective heat transfer, 
the phase front moves upward faster in the case of pure paraffin. It should be noted that the maximum value of average Nusselt number is observed for the case of $\Phi=0$.

\section{Acknowledgements}

This work was conducted as a government task of the Ministry of Education and Science of the Russian Federation (Project Number 13.9724.2017/8.9).

\section{References}

1. C.J Ho, J.Y. Gao, Int. J. Heat Mass Transfer 62, (2013).

2. S.S. Sebti, M. Mastiani, H. Mirzaei, A. Dadvand, S. Kashani, S.A. Hosseini, Applied Physics \& Engineering 14 (2013).

3. L.-W. Fan, Z.-Q. Zhu, Y. Zeng, Q. Lu, Z.-T. Yu, Int. J. Heat Mass Transfer 79 (2014).

4. N.S. Dhaidan, J.M. Khodadadi, T.A. Al-Hattab, S.M. Al-Mashat, Int. J. Heat Mass Transfer 66 (2013).

5. R.S. Vajjha, D.K. Das, Int. J. Heat Mass Transfer 52 (2009).

6. A. Ebrahimi, A. Dadvand, Alexandria Engineering Journal 54 (2015).

7. M. Bayat, M.R. Faridzadeh, D. Toghraie, Thermal Science and Engineering Progress 5 (2018).

8. Y.M.F. El Hasadi, J.M. Khodadadi, J. Heat Transfer 135 (2013).

9. M. Jourabian, M. Farhadi, A.A.R. Darzi, Applied Mathematical Modelling 37 (2013).

10. M. Jourabian, M. Farhadi, K. Sedighi, Computers and Mathematics with Applications 67 (2014).

11. A.J. Chamkha, A. Doostanidezfuli, E. Izadpanahi, M. Ghalambaz, Advanced Powder Technology 28 (2017).

12. N.S. Bondareva, M.A. Sheremet, Thermophysics and Aeromechanics 23 (2016).

13. N.S. Bondareva, M.A. Sheremet, Int. J. Mechanical Sciences 134 (2017).

14. N.S. Bondareva, M.A. Sheremet, Int. J. Thermal Sciences 115 (2017). 